\title{
Dynamic effects of the Ebbinghaus illusion in grasping: Support for a planning/control model of action
}

\author{
SCOTT GLOVER and PETER DIXON \\ University of Alberta, Edmonton, Alberta, Canada
}

\begin{abstract}
A distinction between planning and control can be used to explain the effects of context-induced illusions on actions. The present study tested the effects of the Ebbinghaus illusion on the planning and control of the grip aperture in grasping a disk. In two experiments, the illusion had an effect on grip aperture that decreased as the hand approached the target, whether or not visual feedback was available. These results are taken as evidence in favor of a planning/control model, in which planning is susceptible to context-induced illusions, whereas control is not. It is argued that many dissociations between perception and action may better be explained as dissociations between perception and on-line control.
\end{abstract}

The distinction between the premovement planning of an action and its on-line control has a long history (e.g., Jeannerod, 1988; Keele \& Posner, 1968; Woodworth, 1899). Here, we demonstrate that the earlier portions of a grasping movement are more affected by the Ebbinghaus illusion than are the latter portions. These results provide further support for a planning/control model (Glover, 2001; Glover \& Dixon, 2001a, 2001b, 2001d) in which planning is more susceptible to illusions than control. The results do not support a perception/action model (e.g., Aglioti, DeSouza, \& Goodale, 1995; Bridgeman, 1999; Bridgeman, Peery, \& Anand, 1997; Goodale \& Milner, 1992; Milner \& Goodale, 1995), in which both planning and control are thought to be less susceptible to illusions than are perceptions.

Woodworth (1899) was the first to demonstrate the distinction between the premovement planning and the on-line control of action. Woodworth found that when participants were asked to draw lines of particular lengths, they required sufficient time to correct the actions on line. For example, accuracy was worse at a drawing rate of $400 \mathrm{msec}$ per line than at slower rates. Furthermore, if the linedrawing task was done without vision, participants' performance at all speeds was just as poor as when the task was done quickly with vision. Woodworth reasoned from this that actions were composed of two stages: an "initial impulse" stage that reflected the premovement planning of the action and a subsequent "current control" stage that reflected the on-line correction of an action via feedback mechanisms.

Correspondence concerning this article should be addressed to S. Glover, Department of Psychology, Pennsylvania State University, 541 Moore Bldg., University Park, PA 16402 (e-mail: srg13@ psy.edu).
Since Woodworth's (1899) seminal study, much research has gone into characterizing these two stages of action (e.g., Abrams \& Pratt, 1993; Elliot, Binsted, \& Heath, 1999; Flash \& Henis, 1991; Keele \& Posner, 1968; Khan, Franks, \& Goodman, 1998; Meyer, Abrams, Kornblum, Wright, \& Smith, 1988; Pratt \& Abrams, 1996), and some distinctions between the two stages have been elucidated. For example, planning appears to be a relatively slow and deliberate process. The minimum time required to initiate a movement has been found to be around $250 \mathrm{msec}$ (Stark, 1968). Conversely, on-line control appears to operate relatively quickly. In contrast to the $400-\mathrm{msec}$ lag between planning and control stages hypothesized by Woodworth on the basis of his original study, the benefits of visual and proprioceptive feedback during on-line control have more recently been found to occur in as little as $70-150 \mathrm{msec}$ (e.g., Evarts \& Vaughn, 1978; Lee \& Tatton, 1975; Paulignan, MacKenzie, Marteniuk, \& Jeannerod, 1991; Smeets, Erkelens, \& van der Gon, 1990; Zelaznik, Hawkins, \& Kisselburgh, 1983).

We have hypothesized that another difference between planning and control may be how each is affected by context-induced visual illusions. In the planning/control model (Glover, 2001; Glover \& Dixon, 2001a, 2001b, 2001d), context-induced illusions affect the planning process, but not the on-line control process. We use the term context-induced to refer to distortions that arise owing to the visual context surrounding the target, as opposed to those that result from other types of optical effects, such as some perceptual constancy mechanisms (e.g., saccadic suppression; Goodale, Pelisson, \& Prablanc, 1986; Prablanc \& Martin, 1992), exposure aftereffects (e.g., the McCollough effect; McCollough, 1965), or the wearing of light-refracting devices (e.g., prism goggles; Redding \& Wallace, 1994, 1997). 
The different effects of visual illusions on the planning and control of actions are assumed to be due to the fact that each stage of action uses its own visual representation. The crucial difference between the two representations pertains to the visual context surrounding the target. On the one hand, the visual representation used in planning incorporates the visual context surrounding the target. Information about the context is crucial in planning movements that avoid obstacles and/or depend on affordances involving the context. On the other hand, the visual representation used in control operates relatively independently of the context. This allows it to operate quickly and with a high level of accuracy by focusing its computational power solely on the target itself. As a consequence of the differential role of the context in the two representations, planning is affected by context-induced illusions, whereas control is largely immune to such effects.

This model provides a ready account of the reported pattern of illusion effects on action. For example, parameters of action that are the most affected by context-inducedillusions are also those that likely reflect planning processes, such as reaction time (Smeets \& Brenner, 1995), movement time (Gentilucci, Chieffi, Daprati, Saetti, \& Toni, 1996; Smeets \& Brenner, 1995; van Donkelaar, 1999), posture choice (Glover \& Dixon, 2001a), and initial hand orientation (Glover \& Dixon, 2001a, 2001b, 2001d). Conversely, parameters of action that are less affected by illusions are those that occur at or near the end of the movement and are thus most likely to be influenced by on-line control processes. These include pointing accuracy (Bridgeman, Gemmer, Forsman, \& Huemer, 2000; Bridgeman, Lewis, Heit, \& Nagle, 1979; Bridgeman et al., 1997), maximum grip aperture (Aglioti et al., 1995; Brenner \& Smeets, 1996; Daprati \& Gentilucci, 1997; Haffenden \& Goodale, 1998; S. R. Jackson \& Shaw, 2000; Otto-de Haart, Carey, \& Milne, 1999; but see Franz, Gegenfurtner, Bülthoff, \& Fahle, 2000; Pavani, Boscagli, Benvenuti, Rabuffetti, \& Farne, 1999), and final hand orientation (Glover \& Dixon, 2001a, 2001b, 2001d).

An alternative to the planning/control model is the perception/action model (Aglioti et al., 1995; Goodale \& Milner, 1992; Haffenden \& Goodale, 1998; Milner \& Goodale, 1995). This model is based on a dorsal-stream/ ventral-stream dichotomy in the visual processing pathways of the macaque brain (Mishkin, Ungerleider, \& Macko, 1983) and its putative homologue in the human brain. According to the perception/action model, illusion effects on action are often small or nonexistent because actions typically use a visual representation that only weakly encodes the context surrounding the target. This context-independent action representation is thought to allow the motor system to accurately compute the characteristics of the target for both the planning and the control of actions. Conversely, the perception module is thought to include the visual context surrounding the target for the purpose of helping to identify objects and attach meaning to them. According to the perception/action model, actions will be affected by illusions only when the perception and action modules interact prior to movement initiation. These interactions are said to be necessary when information related to the target's identity (such as its function, weight, or texture) is required in order to carry out an action.

The perception/action model makes many predictions similar to those of the planning/control model and is thus also supported by many of the same results (e.g., Aglioti et al., 1995; Bridgeman et al., 1997; Daprati \& Gentilucci, 1997; Haffenden \& Goodale, 1998). However, other results are more consistent with the planning/control model than with the perception/action model. For example, it has been found that illusion effects on action are smaller when visual feedback is available to participants, as compared with when it is not available (Gentilucci et al., 1996; Glover \& Dixon, 2001c; Westwood, Heath, \& Roy, 2000). This supports the notion, inherent in the planning/control model, that on-line control processes are responsible for the small illusion effects on actions. Conversely, any reduction in illusion effects owing to the availability of visual feedback would seem to be inconsistent with the perception/ action model, because both the planning and the control of actions are held to be relatively immune to illusions.

Another line of evidence in favor of the planning/control model comes from studies examining the dynamic illusion effect (Glover \& Dixon, 2001a, 2001b, 2001d). In these studies, we measured the effect of an orientation illusion on the orientation of the hand as participants reached out to grasp a bar. We found that the effect of the illusion on the orientation of the hand was large early in the reach but decreased to near zero by the end of the reach, allowing participants to grasp the bar without difficulty. According to the planning/control model, the effect of the illusion on hand orientation decreased because the illusion affected the initial planning of the movement, but not its subsequent control. In contrast, a dynamic illusion effect would not be predicted by a perception/action model. According to this model, the illusion should have a small and consistent effect throughout the reach, because both planning and control make use of a context-independent action representation.

\section{Overview of the Present Study}

As a further test of the planning/control and perception/ action models, we here measured the effects of the Ebbinghaus size-contrast illusion on grasping. Participants reached out and grasped a target disk lying flat in front of them, using a pincer grasp employing the thumb and forefinger. During such an action, the grip aperture (i.e., the distance between the thumb and the forefinger) first increases to a maximum somewhat larger than the actual size of the target, then decreases as the hand closes around the target (e.g., Jakobson \& Goodale, 1991; Jeannerod, 1984; Marteniuk, MacKenzie, Jeannerod, Athenes, \& Dugaas, 1987; Wing, Turton, \& Fraser, 1986). In order to study the effect of a context-induced illusion on this action, the apparent 

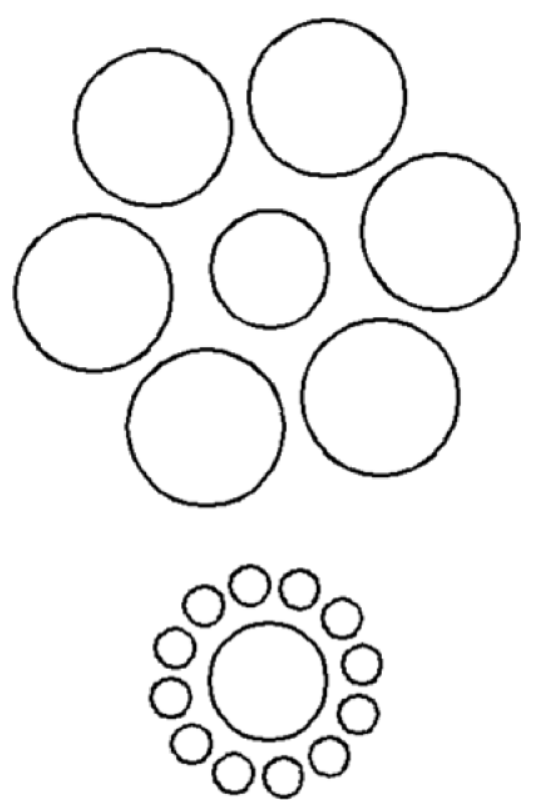

Figure 1. The Ebbinghaus illusion used in the present study. The targets presented in the middle of each display are the same size. On the top, the contextual circles are large, making the target appear slightly smaller than it really is. On the bottom, the contextual circles are small, making the target appear slightly larger than it really is.

size of the target disk was manipulated by surrounding the target with circles that were either larger or smaller than the target (Figure 1). The resulting size-contrast illusion is known as the Ebbinghaus (or Titchener) illusion (e.g., Coren \& Girgus, 1978; Coren \& Miller, 1974) and has been extensively studied with regard to its effects on action (Aglioti et al., 1995; Franz et al., 2000; Haffenden \& Goodale, 1998; Marotta, DeSouza, Haffenden, \& Goodale, 1998; Pavani et al., 1999). The Ebbinghaus illusion owes its effects to the relation between the target and the contextual figures. Specifically, when the contextual figures are larger than the target, the target appears to be smaller than it really is (Figure 1, top). Conversely, when the contextual figures are smaller than the target, the target appears larger than it really is (Figure 1, bottom).

Past studies have often found that the effect of the Ebbinghausillusion on grasping is dissociable from its effects on perceptual judgments (e.g., Aglioti et al., 1995; Haffenden \& Goodale, 1998; but see Franz et al., 2000; Marotta et al., 1998; Pavani et al., 1999). For example, Aglioti et al. found that the effect of the Ebbinghaus illusion on the maximum grip aperture (i.e., the maximum distance between the thumb and the finger in grasping) was smaller than its effect on perceptual judgments indexed through the method of comparison. A follow-up study by Haffenden and Goodale replicated this finding under visual open-loop conditions, suggesting that the small effect on maximum grip aperture did not depend on visual feedback. These results have been taken as evidence in favor of a perception/action model (Aglioti et al., 1995; Haffenden \& Goodale, 1998; Milner \& Goodale, 1995).

However, this pattern of results is also consistent with the planning/control model. The principal dependent variable in these studies, the maximum grip aperture, usually does not occur until well into the second half of the movement (Jakobson \& Goodale, 1991; Jeannerod, 1984; Marteniuk et al., 1987; Wing et al., 1986). Consequently, there is ample time in which on-line control mechanisms could operate (see Bock \& Jungling, 1999; Castiello, Bennett, \& Stelmach, 1993; Paulignan, Jeannerod, MacKenzie, \& Marteniuk, 1991). In our view, maximum grip aperture may not only represent how an action is planned, but also how it is controlled.

In the present research, we measured the effects of the Ebbinghaus illusion on grasping at a range of points throughout the reach, rather than at the point of the maximum grip aperture only. Using this approach, we were able to distinguish the effects of the illusion on how the grasps were planned (evident early in the movements) from its effects on how the grasps were controlled (evident later in the movements).

\section{EXPERIMENT 1}

Experiment 1 tested the predictions of the planning/ control and perception/action models by comparing the effects of the Ebbinghausillusion at several points during the reach. If the planning/control model is correct, the effect of the Ebbinghaus illusion on grip aperture should be large early in the reach but decline as the hand approaches the target. This would reflect the use of a context-dependent visual representation in planning and the use of a contextindependent visual representation in on-line control. Conversely, if the perception/action model is correct, the effect of the illusion on grip aperture should be small and fairly stable throughout the course of the reach. This would reflect the use of a context-independent action representation in both the planning and the control of action.

\section{Method}

Participants. Twelve University of Alberta undergraduates participated in the experiment in return for course credit. All 12 participants were right-handed and had normal or corrected-to-normal vision. All the participants were naive as to the exact purpose of the study.

Apparatus. The participant sat on an adjustable chair at a $100 \times$ $60 \mathrm{~cm}$ table, behind a $20 \times 80 \mathrm{~cm}$ two-way mirror positioned approximately $10 \mathrm{~cm}$ from the participant's eyes. The top of the two-way mirror was rotated $15^{\circ}$ away from the participant in the frontal plane. The participant's vision above and around the table top was restricted by the testing apparatus, but he or she was able to see the table top, including his or her reaching hand and arm, throughout each trial. Head and eye movements were not restricted.

A system of lights was used to either block or allow vision through the two-way mirror. One light was positioned above the table at a height of $52 \mathrm{~cm}$, close to the participant's end of the table. This light remained lit throughout the experiment. Two floodlights were set on the table to the left and right sides of the workspace, and two more floodlights were positioned above the participant, to the 
left and right. To allow vision through the two-way mirror, the lights on the table were lit, and the lights above the participant were extinguished. To block vision through the mirror, the lighting arrangement was reversed. When the table top was visible, its illumination was $0.9 \mathrm{~cd} / \mathrm{m}^{2}$ (measured from the participant's side of the two-way mirror). When the table top was not visible, the participant saw only the reflection of the ceiling in the two-way mirror.

U.S. letter-sized $(27.9 \times 21.6 \mathrm{~cm})$ pieces of paper with the Ebbinghaus circles illusion printed on them were placed on the table in the center of the participant's field of view. The paper occupied $29.9^{\circ}$ of visual angle in the depth plane and $23.1^{\circ}$ of visual angle in the horizontal plane. The large-circles version of the illusion (Figure 1, top) was made up of 6 evenly spaced circles (41-mm diameter, or $4.3^{\circ}$ of visual angle) surrounding the center of the paper. The edge of each large circle was $25 \mathrm{~mm}\left(2.7^{\circ}\right.$ of visual angle $)$ from the center of the paper. The small-circles version of the illusion (Figure 1, bottom) was made up of 12 evenly spaced circles $\left(1.0-\mathrm{mm}\right.$ diameter, or $1.1^{\circ}$ of visual angle) surrounding the center of the paper. The edge of each small circle was $20 \mathrm{~mm}\left(2.1^{\circ}\right.$ of visual angle) from the center of the paper.

Small white plastic disks, ranging in $2-\mathrm{mm}$ increments from $26 \mathrm{~mm}\left(2.8^{\circ}\right.$ of visual angle $)$ to $34 \mathrm{~mm}\left(3.6^{\circ}\right.$ of visual angle), replaced the target circles in the middle of the paper. Each disk was $3 \mathrm{~mm}$ thick. The outside rims of the disks were painted black so that the disks would resemble the contextual circles as closely as possible. The center of the disks was approximately $43 \mathrm{~cm}$ from the participant's midsection. A $1 \times 5 \mathrm{~cm}$ cylindrical starting bar was taped to the table, using surgical adhesive tape, so that its length was parallel with the participant's frontal plane. The center of the starting bar was approximately $23 \mathrm{~cm}$ from the participant's midsection and exactly $20 \mathrm{~cm}$ from the center of the disks.

The participant wore two infrared light-emitting diodes (ireds), one taped to the mediodorsal surface of the thumb, roughly halfway between the interphalangeal joint and the tip of the thumb, and the other to the laterodorsal surface of the index finger, roughly halfway between the proximal interphalangeal and the distal interphalangeal joints. If one imagines a participant's hand lying on a table with the palm facing down, the ireds were worn on the skin near the base of the nails of the thumb and forefinger, on the side to the right of the nail on the thumb and to the left of the nail on the finger. The ireds were attached with wires to a computer, and the loose wire was fastened to the participant's forearm with enough play that the participant could move his or her arm and hand freely. None of the participants reported feeling any restriction or discomfort while wearing the ireds.

The table top was monitored with an infrared video camera fixed to a stable mount at a height of $70 \mathrm{~cm}$ above the table. The two ireds were alternately illuminated at $60 \mathrm{~Hz}$, and the position of the lit diode was detected electronically every video frame and recorded using an IScan tracking system. The IScan system output the position of the lit diode to a computer at $60 \mathrm{~Hz}$, with a nominal resolution of $1.2 \mathrm{~mm}$ horizontally and $2.2 \mathrm{~mm}$ in depth. In order to assess the precision of the system for monitoring movements, we used a method adapted from Haggard and Wing (1990). Two diodes were attached to a plastic card $8 \mathrm{~cm}$ apart. The card was swept systematically across different portions of the camera field of view at velocities comparable with those during reaching (about $0.5 \mathrm{~m} / \mathrm{sec}$ ). The distance between the diodes was reconstructed from the system output, with a standard deviation of $0.60 \mathrm{~mm}$ laterally and $1.04 \mathrm{~mm}$ in depth. There were no measurable deviations from linearity in the workspace using the IScan system.

Experimental procedure. The participants were required to begin each trial by pinching the starting bar with the thumb and forefinger, keeping the other fingers curled underneath the hand. A trial began when the computer, at the experimenter's instigation, simultaneously extinguished the lights behind the screen and lit the lights on the table, making the stimulus array visible through the two-way mirror. The participants were required to reach out and pick up the disk, using the thumb and the forefinger, and then set the disk down on the half of the table top nearest themselves. Instructions to the participants did not emphasize speed. Six randomly determined practice trials were run before the test trials to allow the participants to familiarize themselves with the task.

Two contextual circles conditions were used. In half the trials, the context consisted of the large circles; in the other half, it consisted of the small circles. For each context condition, five disk sizes were used: $26,28,30,32$, and $34 \mathrm{~mm}$. In each of two blocks, the participants received seven repetitions of each context and disk combination, for a total of 120 trials. The participants were allowed a brief rest between blocks if they so desired.

Data analysis. Data were analyzed by passing the position data from the two ireds through a custom filter. The onset of each reach was set at the time when the movement velocity of the thumb exceeded $0.10 \mathrm{~m} / \mathrm{sec}$. The offset of the reach was set at the time when the thumb ceased to move in a forward direction. The thumb was used for determining velocity, because most of the change in grip aperture during a pincer grasp is due to flexion or extension of the finger; as a consequence, the thumb provides a relatively stable index of the overall position of the hand during the reach (Haggard \& Wing, 1997; Wing \& Fraser, 1983; Wing et al., 1986). Grip aperture was computed for each sampled position from reach onset to offset. For each measurement of grip aperture, the position of the ired that was not lit during that frame was interpolated between the position measurements for the preceding and the following frames. Measurements were omitted when they implied a movement velocity greater than $1.0 \mathrm{~m} / \mathrm{sec}$ or when the positions were outside the normal range of movement. Positions for these omitted observations were estimated by interpolation. Trials were excluded if either the reaction time or the movement time was less than $250 \mathrm{msec}$ or greater than $1,500 \mathrm{msec}$ or if the starting position was outside a normal range. Apart from 1 participant who was excluded owing to poor recording, $96.6 \%$ of the trials from 11 participants were included in the final analysis.

On completion of the filtering process, the data for each trial were time normalized. This involved estimating the position and grip aperture by interpolation for 21 equally spaced points in time ranging from the onset of the movement to its offset. Each time slice thus represented $5 \%$ of the duration of the movement. The normalized trials for each background and disk condition were averaged for each participant.

The magnitude of the illusion effect on grip aperture was expressed relative to the effect of the (veridical) disk size. First, for each time slice, the ratio of disk size to grip aperture was computed, averaging over participants and context condition. This size-aperture ratio represents the extent to which the grip varied with disk size at each time slice. Generally, the ratio starts out small at the beginning of the reach and increases as the hand approaches the target disk. Second, the raw effect of the illusion was computed for each subject and time slice by subtracting the grip aperture measured with the small-circles context from that with the large-circles context. Finally, the scaled illusion effect for each participant and time slice was calculated by dividing the raw illusion effect by the size-aperture ratio for that time slice. The scaled illusion effect represents the magnitude of the illusion effect relative to the extent to which grip aperture is tuned to the size of the disk. In other words, the scaled effect provides a measure of how much of a difference in actual disk size is represented by the raw illusion effect. The scaled effect of the illusion on grip aperture was used in the subsequent analysis.

Two issues were assessed statistically. First, was there an overall effect of the context-induced illusion on grip aperture - that is, was the average scaled illusion effect greater than zero? Second, did the scaled illusion effect change over time? These questions were ad- 


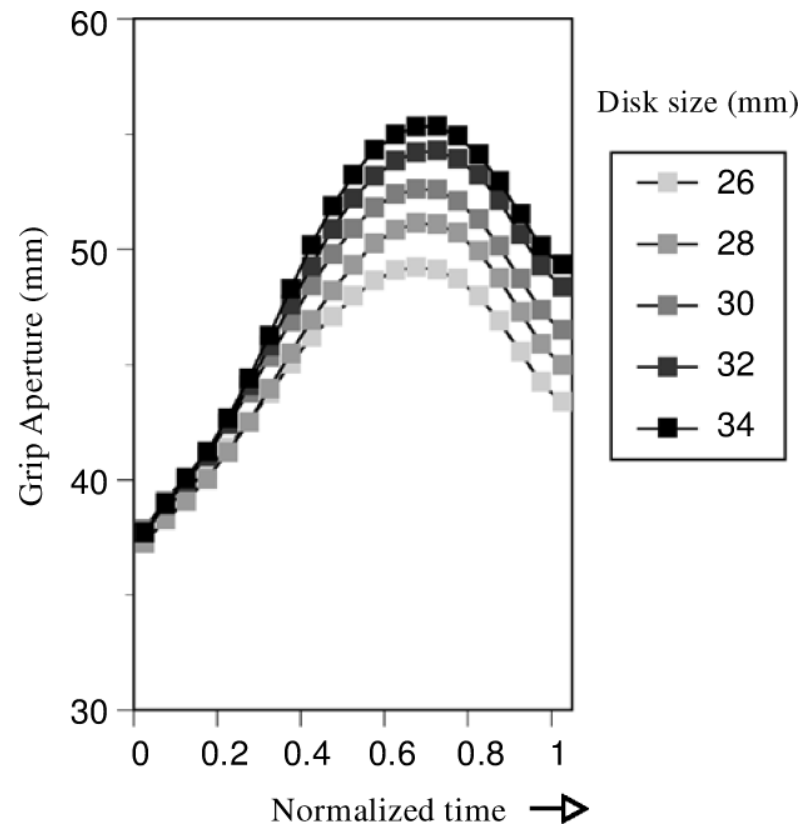

Figure 2. The effect of each of the five disk sizes on grip aperture in Experiment 1. Grip aperture is represented on the $y$-axis, normalized time on the $x$-axis.

dressed by fitting a series of nested linear models to the data for the scaled illusion effect. In the first model, it was assumed that the mean illusion effect was zero at all points in time- that is,

$$
\mu_{t}=0 \text {. }
$$

In the second model, it was assumed that there was a constant effect of the illusion-that is,

$$
\mu_{t}=\alpha .
$$

In the third model, an effect of time was added:

$$
\mu_{t}=\alpha+\beta_{t}
$$

where

$$
\sum_{t} \beta_{t}=0
$$

The relative fits of these three models were evaluated by computing maximum-likelihood ratios. The likelihood ratio indicates the likelihood of the data given the best fit of one model divided by the likelihood of the data given the best fit of the other. The likelihood ratio provides an index of the strength of the evidence for one model relative to the other (Goodman \& Royall, 1988). The likelihood of the data for a linear model with normally distributed error is closely related to the residual variance not predicted by the model and can be computed as follows (Dixon \& O'Reilly, 1999):

$$
\lambda=\left(\frac{S S E_{2}}{S S E_{1}}\right)^{\frac{n}{2}},
$$

where $S S E_{1}$ and $S S E_{2}$ reflect the fit of the two models and $n$ is the number of independent observations. Because the scaled illusion effect is essentially a difference score, we assumed that the observed effects for each subject at each point in time were independent. We adopt the heuristic that a likelihood ratio of 10 or greater indicates relatively clear evidence for one model over the other. Under many circumstances, the likelihood ratio thus obtained is closely related to the $p$ value found by testing the hypothesis of no effect (Dixon, 1998), and, if one were to do null hypothesis significance testing, the null hypothesis would generally be rejected if the likelihood ratio were 10 or greater.

\section{Results}

Figure 2 shows the effect of disk size on grip aperture over time for the 11 participants who produced usable data. It can be seen in Figure 2 that the grip aperture gradually became more dependent on the disk size as the movements progressed. The maximum grip aperture occurred at around $65 \%$ of the movement, comparable with what has been typically found (Jakobson \& Goodale, 1991; Jeannerod, 1984; Marteniuk et al., 1987; Wing \& Fraser, 1983).

The scaled difference in grip aperture between the two contextual circles conditions is shown in Figure 3. The data were analyzed by comparing the scaled effect of the context at $t=0.4,0.6,0.8$, and 1.0 (i.e., at the completion of $40 \%, 60 \%, 80 \%$, and $100 \%$ of the reach). The scaled effect for the times prior to $t=0.4$ were excluded because the ratio relating grip aperture to disk size was very small early in the reach and, as a consequence, the scaled illusion effect tended to be excessively variable at those times. Only four points were used in the analysis to reduce the potential for dependence between observations at successive points in time. It can be seen in Figure 3 that there was a consistent decline in the effect of the illusion from $t=0.4$ onwards, and this was also supported by the statistical analysis. The likelihood ratio comparing a model that included a constant effect of the illusion with a null model that included only an effect of participants was $\lambda>1,000$. In other words, the data were more than 1,000 times as likely on the assumption that an effect of the illusion was present than on the assumption that there was no effect of the illusion. The likelihood ratio comparing a full model that included an

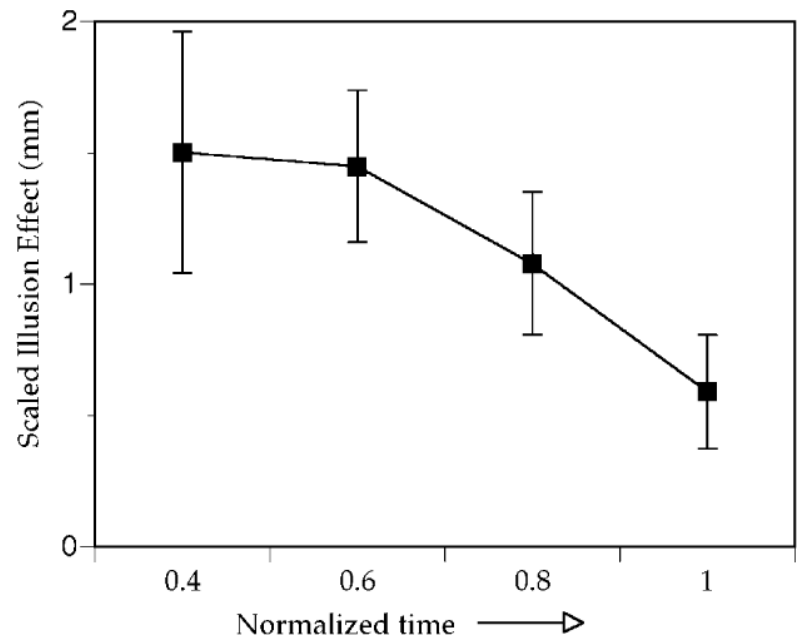

Figure 3. The scaled illusion effect on grip aperture in Experiment 1 . The effect is shown for the four points in normalized time used in the data analysis. Error bars represent standard errors of the mean. 
effect of the illusion and an effect of time (consistent with the planning/control model) with a model that included an effect of the illusion but no effect of time (consistent with the perception/action model) was $\lambda=14.1$. In other words, the data were more than 14 times as likely on the assumption that an effect of time was present than on the assumption that there was no effect of time.

Mean reaction time across participants was $613 \mathrm{msec}$ (range, 389-929 msec). Mean movement time across participants was $570 \mathrm{msec}$ (range, 442-904 msec). There was no indication that the illusion or the size of the disks had effects on either reaction times or movement times.

\section{Discussion}

The fact that the effect of the Ebbinghausillusion on grip aperture decreased as the hand approached the target in Experiment 1 is consistent with the planning/control model. In this model, it is held that actions are planned using a context-dependentrepresentation that is susceptible to illusions but are controlled using a context-independent representation that is immune to visual illusions. The data from Experiment 1 are less consistent with a perception/ action model, in which it is held that actions are both planned and controlled using a context-independent representation that is immune to illusions and separate from that used in perception. The dynamic illusion effect might be explained within the perception/action model by assuming that the two modules interact in the course of the action, with the perception module making a substantial contribution to the planning of the movement. However, since there would appear to be no a priori necessity for an interaction of this sort, such an assumption would tend to undermine the distinction between perception and action that motivates this approach.

\section{EXPERIMENT 2}

Although Experiment 1 revealed a dynamic effect of the Ebbinghaus illusion on grasping, showing that the effects of this illusion on action could be corrected in flight, the results do not indicate which sources of information were crucial to the correction process. Information from any or all of visual feedback, proprioception, or efference copy may have contributed. The aim of Experiment 2 was thus to dissociate the contributions of visual and nonvisual sources of information in the correction process by including a novision condition in which participants reached without vision of their hand or the target. A vision condition like that used in Experiment 1 was also included for comparison.

It is commonly understood that visual feedback aids in the accuracy of reaching movements (Elliot et al., 1999; Jeannerod, 1988; Keele \& Posner, 1968; Woodworth, 1899). However, on-line adjustments have also been found to occur in the absence of visual feedback (e.g., Goodale et al., 1986; Khan et al., 1998; Prablanc \& Martin, 1992), demonstrating that nonvisual sources of information also play an important role during on-line control. For example, the loss of proprioception through pathology has detrimental effects on motor performance, suggesting that proprioception is important for the control of action (Gentilucci, Toni, Chieffi, \& Pavesi, 1994; G. M. Jackson et al., 2000; Jeannerod, Michel, \& Prablanc, 1984; Lee \& Tatton, 1975; Sainburg, Poizner, \& Ghez, 1993). A role for efference copy may also be present, on the basis of studies that show that localization of an actively moved limb (i.e., one the participant moved himself or herself) is superior to localization of a passively moved limb (i.e., a limb moved by the experimenter; Eklund, 1972; Paillard \& Brouchon, 1968). Thus, there is evidence that both visual and nonvisual sources of information contribute to on-line control.

On the basis of this, we predict that in the planning/ control model, illusion effects should decrease over time whether or not visual feedback is available, although the reduction in the illusion effect over time may be less substantial in a no-vision condition than in a vision condition. In contrast, the perception/action model includes the premise that actions are planned and controlled using a common visual representation that is less susceptible to illusions than are perceptions. This would seem to imply that removing vision should not affect the illusion effect on grasping. Furthermore, as was discussed in connection with Experiment 1, according to a proponent of the perception/action model, the effect of the illusion should not change over the course of the reach, whether or not vision is available.

Experiment 2 also included a test of the perceptual effects of the Ebbinghaus illusion. It is possible that the effect of the illusion in our setup may have been different from that in previous studies because the stimulus display was viewed from a seated position (rather than standing) and was thus slightly foreshortened. A perceptual task would thus provide measurements that could be directly compared with the effect observed in grasping.

\section{Method}

Participants. Sixteen University of Alberta undergraduates participated in the experiment in return for course credit. All 16 participants were right-handed and had normal or corrected-to-normal vision. All the participants were naive as to the exact purpose of the study.

Apparatus. The apparatus was identical to that used in Experiment 1.

Reaching task procedure. The participants performed two blocks of reaching trials and one block of perception trials. The reaching trials were divided into one vision and one no-vision block. The order of blocks was counterbalanced across participants, with the perception block coming directly before the reaching blocks in half of the participants and directly after the reaching blocks in the other half of the participants. The procedure for reaching trials in the vision block was identical to that used in Experiment 1.

The no-vision trials differed from the vision trials in the following respects: In the no-vision trials, the participants were first given a preview of the display. After $2 \mathrm{sec}$, the participants' view of the table top (including their hands) was blocked, and a tone signaled the participants to reach. Other than the 2-sec preview, the tone, and the lack of vision during the reach, the task was identical in all respects to that in the vision trials. The participants were given 6 randomly de- 
termined practice trials prior to beginning the vision task and 10 randomly determined practice trials prior to beginning the no-vision task.

The context and disk conditions used in the reaching task were identical to those used in Experiment 1, except that only six repetitions of each condition were used in each of the vision and no-vision blocks. This resulted in 60 trials in each of the two reaching blocks, for 120 trials in total. The participants were allowed a brief rest between the two blocks of reaching trials.

Perception task procedure. The setup for the perception task was identical to that for the reaching tasks, but here the participant was required to match the size of the disks, using the thumb and forefinger. Trials began with the experimenter's setting up the display with the participant's vision blocked. Once the display was set up, the lights were switched, and the participant had to lift his or her hand up off the starting bar and indicate the size of the disk, using the thumb and forefinger. He or she was instructed not to move the hand toward the disk (which could have allowed him or her to interpose the hand in the line of sight of the disk). A trial continued for $5 \mathrm{sec}$, at which time a tone sounded. The participant was then required to return the hand to the starting position and await the next trial.

In half the perception trials, the context consisted of the large circles; in the other half, it consisted of the small circles. For each context condition, three disk sizes were used: 26,30 , and $34 \mathrm{~mm}$. The participants received two repetitions of each context and disk combination, for a total of 2 repetitions $\times 3$ disks $\times 2$ contexts $=12$ trials. The order of presentation was random.

Data analysis. Data from the reaching trials were analyzed in a fashion identical to that in Experiment 1. A total of $94.5 \%$ of the reaching trials from 15 of the participants were included in the final analysis. One participant was excluded owing to recording failure.

Data from the perception trials were analyzed by dividing the recordings into 11 measurements spaced at $100-\mathrm{msec}$ intervals over the final $1,000 \mathrm{msec}$ of each trial. Distance measures from these 11 measurements were averaged, and the mean distance was computed for each trial for each participant. Distances across the two repetitions were averaged. Poor recording resulted in the exclusion of $5.6 \%$ of the trials. However, at least one trial for each condition for each participant was available, and no participants had to be excluded for this reason. The scaled effect of the illusion on perceptual estimates was calculated by dividing the raw illusion effect on manual estimates by the ratio of the effect of disk size on manual estimates.

\section{Results}

Figure 4 shows the effect of disk size on grip aperture over time for both the vision (left) and the no-vision (right) conditions. A clear dependence of grip aperture on disk size was present from the beginning of the movement in both conditions. It is clear from Figure 4 that the grip aperture was generally larger and reached its peak slightly earlier in the no-vision condition than in the vision condition. This replicates findings reported in other studies of grasping without vision (Jakobson \& Goodale, 1991; Jeannerod, 1984).

The scaled illusion effect for the two vision conditions and the four analyzed time slices is shown in Figure 5. The illusion effect in the two vision conditions tended to decrease as the hand approached the target, and this decrease was more evident in the no-vision condition than in the vision condition. When a model that included a constant effect of the illusion was compared with a null model, the likelihood ratio was large $(\lambda>500)$. That is, the data were more than 500 times as likely on the basis of the assumption that the illusion had an effect on grip aperture than on the assumption that the illusion had no effect. When an effect of time was added into this model, the likelihood ratio comparing it with the model that included only the effect

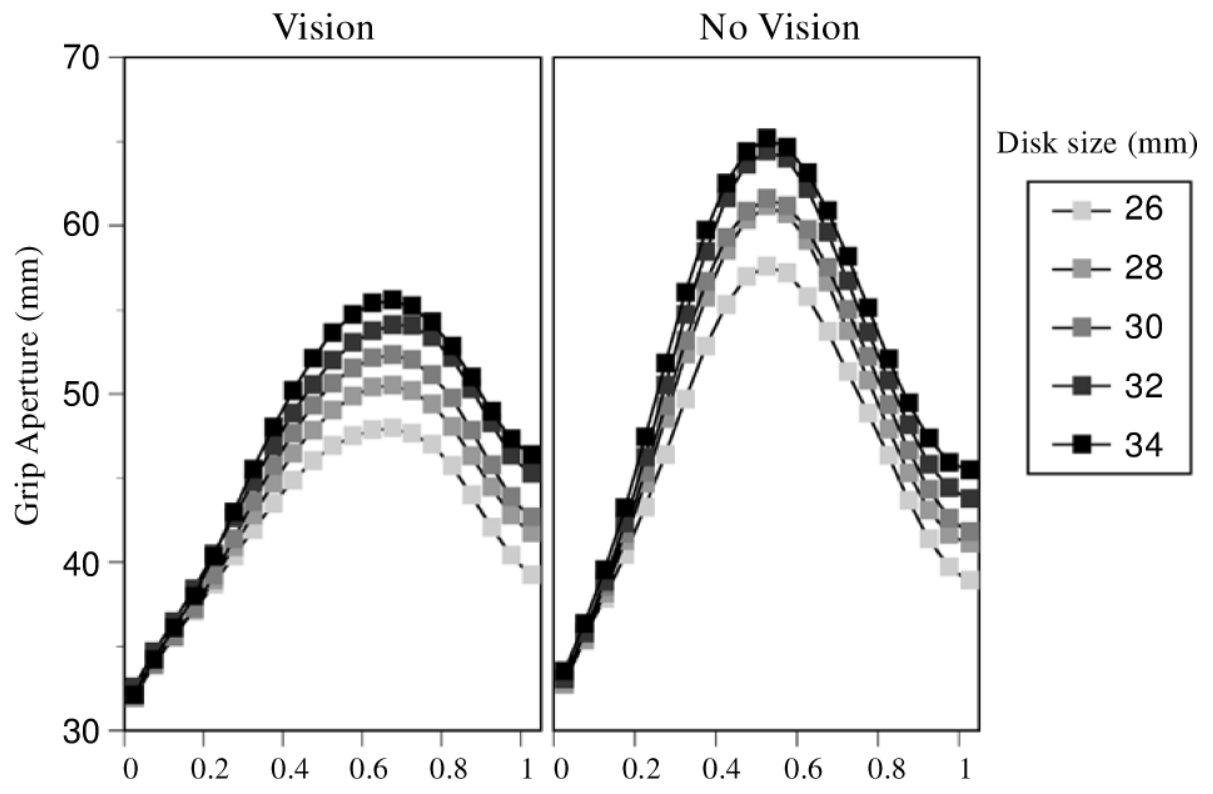

Figure 4. The effect of each of the five disk sizes on grip aperture in the vision (left) and novision (right) conditions of Experiment 2. Grip aperture is represented on the $\boldsymbol{y}$-axis, normalized time on the $x$-axis. 

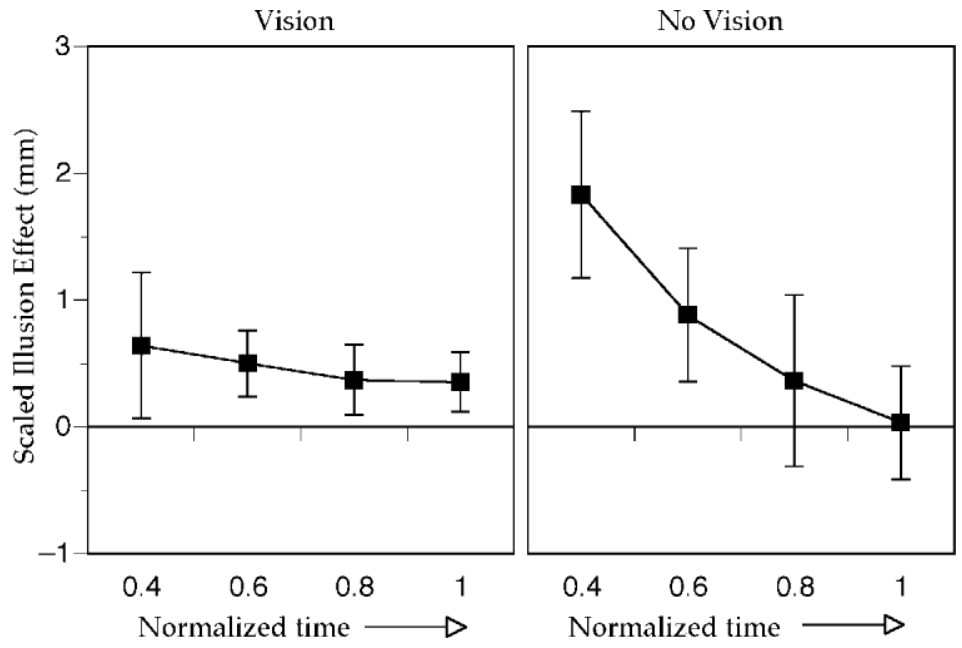

Figure 5. The scaled effect of the illusion on grip aperture in the vision (left) and no-vision (right) conditions of Experiment 2. The effect in each condition is shown for the four points in normalized time used in the data analysis. Error bars represent standard errors of the mean.

of the illusion was also large $(\lambda=17.0)$, suggesting that the illusion effect decreased as the hand approached the target.

Although the pattern of means shown in Figure 5 suggests an interaction in which the illusion effect early in the reach is larger without vision, the evidence for such an interaction was not compelling. When a full model that added in the effects of vision condition and the interaction of time with vision was compared with a model that included only the effects of the illusion and time, the likelihood ratio was $\lambda=7.0$. To further examine this possibility, we compared the largest illusion effects in the two vision conditions at $t=0.4$. Here, the illusion effect was $0.64 \mathrm{~mm}$ in the vision condition and $1.83 \mathrm{~mm}$ in the no-vision condition. In this case, the $95 \%$ confidence interval for the difference was -1.54 to $3.32 \mathrm{~mm}$ and included 0 . In sum, whereas there was relatively clear evidence for a constant effect of the illusion and time in Experiment 2, there was no strong evidence either for an effect of either vision condition or for an interaction between time and vision.

The effect of the illusion and its interaction with time were also evident in the no-vision condition when considered by itself. For the no-vision data, when a null model was compared with a model that included a constant effect of the illusion, the likelihood ratio was large $(\lambda=32.8)$; when the constant model was compared with a model that included an effect of time, the likelihood ratio was $\lambda=$ 19.3. This constitutes clear evidence that the effect of the illusion was greater than zero, on average, and diminished as the hand approached the disk. A similar analysis of the data in the vision condition by itself revealed evidence for a constant effect of the illusion $(\lambda=26.9)$ but no clear evidence that the effect varied over time $(\lambda=1.2)$. However, this condition was essentially a replication of Experiment 1 , and the pattern of means is generally consistent with the results found previously. Consequently, we attribute the lack of clear evidence for an effect of time to the variability of the data.

Mean reaction time across participants was $534 \mathrm{msec}$ (range, 341-759 msec) in the vision condition and 461 msec (range, 287-686 msec) in the no-vision condition. Mean movement time across participants was $731 \mathrm{msec}$ (range, 564-1,041 msec) in the vision condition and 923 msec (range, 674-1,154 msec) in the no-vision condition. Reaction times were shorter in the no-vision condition than in the vision condition, presumably because the participants in the no-vision condition were better able to anticipate the onset of each trial. Movement times were some-

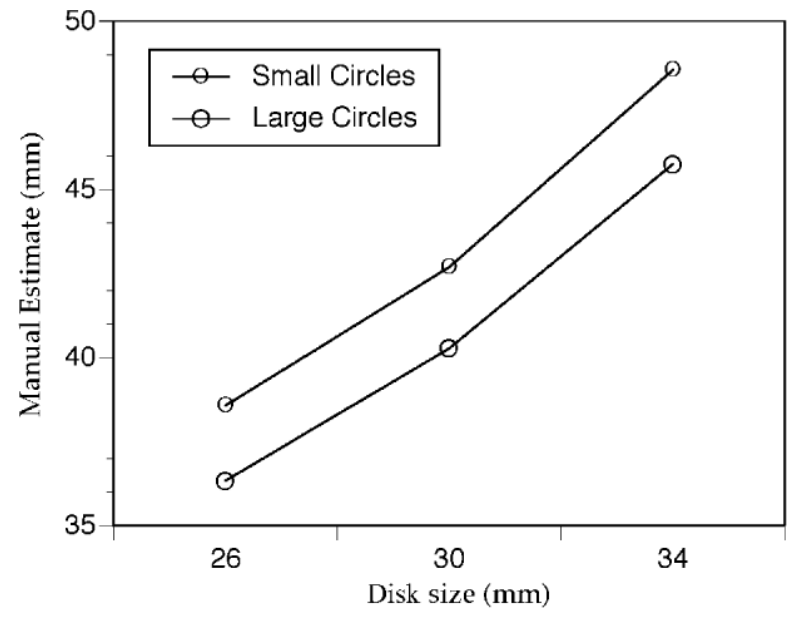

Figure 6. The effects of background condition and disk size in the perceptual matching task of Experiment 2. Estimates made with the small-circles background are represented by the small circles; estimates made with the large-circles background are represented by the large circles. 
what longer in the no-vision condition than in the vision condition, a common finding in studies comparing reaching with and without vision (Connolly \& Goodale, 1999; Jeannerod, 1984). There was no indication that background or disk size affected reaction times or movement times.

Figure 6 shows the results of the perception task in Experiment 2. It is evident from Figure 6 that the participants gave larger estimates for the disks surrounded by small circles than for the disks surrounded by large circles. To assess the effects of the illusion on the estimates, we compared nested linear models. Comparing a model that included an effect of disk with a null model gave a large likelihood ratio $(\lambda>1,000)$. Comparing an additive model that included effects of both disk and illusion with the model that included the effect of disk only also gave a large likelihood ratio $(\lambda>1,000)$. However, comparing the full model that included the effects of disk and illusion and their interaction with the additive model yielded a small likelihood ratio $(\lambda=1.52)$. Thus, there were large effects of both disk and illusion on the perceptual estimates, but there was little indication of an interaction between these two variables.

Figure 7 summarizes the results of the two experiments. Shown are the effects of the illusion on grasping in the early $(t=0.4)$ and late $(t=1.0)$ portions of the reach. Also shown is the illusion effect on perceptually based matching. It can be seen in this figure that the effect of the illusion tends to be smaller in the grasping task (even relatively early in the reaches) than in the perceptual judgment task. Although this result suggests that illusions have smaller effects on actions than on perceptions, other stud- ies have shown that it is more common for the early component of the action to be more affected by an optical illusion than are perceptually based judgments (Glover \& Dixon, 2001a, 2001d, 2002a). In a related study, printed words were found to have large effects on planning but no effects on either control or perceptions (Glover \& Dixon, 2002b).

\section{Discussion}

Experiment 2 replicated the dynamic illusion effect found in Experiment 1. The data in the no-vision condition showed that continuous visual information was not required for the on-line correction of illusion effects on grasping. These data support the planning/control model, in which actions are planned using a context-dependent visual representation but are corrected on line, using a context-independentrepresentation. In contrast, these data do not support a perception/action model in which actions are both planned and executed using a context-independent action representation. According to this model, the illusion should have had a consistently small effect on grip aperture throughout the reach.

The finding that the dynamic illusion effect occurred in the no-vision condition supports the notion that nonvisual mechanisms, such as proprioception and efference copy, can be used to adjust reaching movements on line (Goodale et al., 1986; Khan et al., 1998; Prablanc \& Martin, 1992). The results of Experiment 2 also indicate that continuous visual information does not play an essential role in the correction of the effects of the Ebbinghaus illusion on action. However, this does not discount the role of any visual

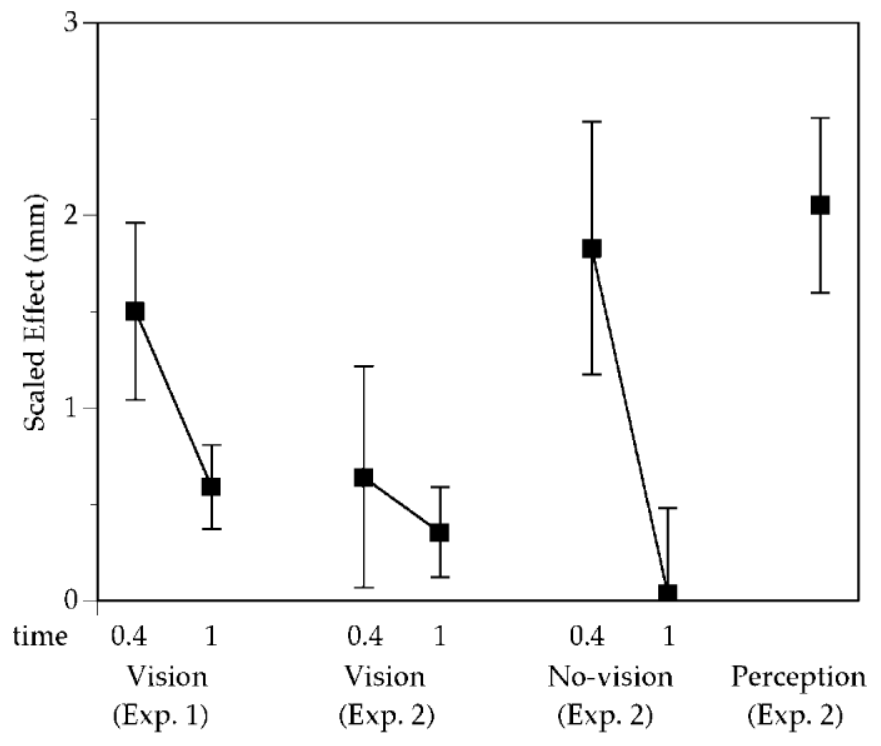

Figure 7. Summary of results of Experiments 1 and 2. Shown are scaled illusion effects for $t=0.4$ and $t=1$ for the grasping task in Experiment 1 and the two conditions of Experiment 2. Also shown is the scaled illusion effect for the perceptual judgment task of Experiment 2. Error bars represent the standard error of the mean. 
information in on-line control: The control system may use stored visual information regarding the target even when there is no feedback loop.

In previous reports, the magnitude of the Ebbinghausillusion on perception has been between 2.25 and $2.50 \mathrm{~mm}$ (Aglioti et al., 1995; Franz et al., 2000; Haffenden \& Goodale, 1998; Pavani et al., 1999), whereas in the present research, the effect was somewhat smaller $(2.05 \mathrm{~mm})$. The smaller perceptual effect may have been due to the participants' slightly foreshortened view of the display. However, despite the smaller perceptual effect, the illusion still had clear effects in both the reaching and the perception tasks. Furthermore, the perceptual effect of the illusion was generally larger than the effect on grip aperture in the last $60 \%$ of the reach. As we mentioned earlier in regard to Figure 7, the illusion effect on grip aperture at $t=0.4$ was nearly as large as its effect on perception, but by the end of the reach the effect on grip aperture had become substantially smaller than the effect on perception.

\section{GENERAL DISCUSSION}

In the present study, we demonstrated a dynamic effect of the Ebbinghausillusion on grasping, in both full-vision and no-vision conditions. This result replicates and extends a similar dynamic effect obtained with an orientation illusion in reaching (Glover \& Dixon, 2001a, 2001b, 2001d). In previous studies, the Ebbinghaus illusion was found to have a smaller effect on the maximum grip aperture than on perceptual judgments of target size (Aglioti et al., 1995; Franz et al., 2000; Haffenden \& Goodale, 1998; Pavani et al., 1999). However, when we measured the effect of the illusion on grip aperture throughout the course of the reach here, we found that the effect of the illusion was large early in the reach and decreased as the hand approached the target. Thus, the relatively small effect of the illusion on maximum grip aperture may be due to the fact that the maximum grip aperture occurs at a relatively late point in the movement. Together, the results of Experiments 1 and 2 suggest that, although the planning of the grasp is affected by the illusion, the on-line control of the grasp is not. This is consistent with the planning/control model, but not with a perception/action model.

The dynamic illusion effect found in the no-vision condition was expected on the basis of past studies that have demonstrated the contributions of proprioception and/or efference copy to on-line control. Although visual feedback may seem to be the most intuitive candidate for the correction of illusion effects on line, the present study suggests that its role under the present circumstances may be relatively minor. However, in other studies using the MüllerLyer illusion, the role of continuous vision of the effectors and targets has been more evident (Gentilucci et al., 1996; Glover \& Dixon, 2001c; Westwood et al., 2000), and a complete understanding of the relative importance of continuous vision in the on-line correction of illusion effects on action requires further research.
Another possible explanation for the dynamic illusion effect observed here and elsewhere is that proprioceptive information becomes more relevant during movement than prior to movement. On this account, illusion effects are corrected on line because proprioception operates as the hand is in flight to correct any error in the planning of the movement. Although this suggestion is intuitively plausible, we note that the present study cannot be used to compare such a notion with the planning/control model.

\section{Other Studies of the Ebbinghaus Illusion and Grasping}

Several authors have argued that the apparent difference between the effects of the Ebbinghaus illusion on action and on perception depends on the manner in which the action and the perception tasks are conducted. For example, in the perception task used by Aglioti et al. (1995), participants compared the apparent sizes of two disks presented with different contextual surrounds, whereas in the grasping task, participants reached out to grasp only one of the disks. Franz et al. (2000) and Pavani et al. (1999) argued that the effect of the illusion may have been reduced in the grasping task because participants were required to attend only to one disk at a time. In support of this interpretation, these authors found little evidence for a difference between grasping and perceptual judgments when both tasks involved only a single target and its surrounding context. Franz et al. concluded that perception and action use a common visual representation and that both are equally affected by the Ebbinghaus illusion, whereas $\mathrm{Pa}-$ vani et al. concluded that their results reflected an interaction between perception and action.

Although the results of Franz et al. (2000) and Pavani et al. (1999) suggest a need for caution when comparing the results of perception and action tasks, their specific concerns would not seem to apply to the manner in which the perception and the action tasks were matched in the Haffenden and Goodale (1998) study. In that study, the effect of the illusion on perception was clearly larger than its effect on the maximum grip aperture, even though the two tasks were appropriately matched by the criterion of Franz et al. and Pavani et al. Similarly, in Experiment 2 of the present study, the effect of the illusion on grip aperture was generally less than its effects on perceptions, even though the two tasks were appropriately matched.

Another factor that may have affected the results in the grasping tasks of the present study was the presence of the annulus (ring of circles) around the target. Haffenden and Goodale (1998) observed that the presence of the annulus may have the effect of presenting "obstacles" to the grasping hand. Generally, the maximum grip aperture was smaller in their study when the hand reached into the center of such an annulus than when no annulus was present. In the present study, it is difficult to imagine how the presence of the annulus would have interacted with the decreasing effect of the illusion on grasping. We would suggest that, being a contextual element, the annulus likely has its ef- 
fects mainly on the planning of the grasp. Indeed, if the annulus were the sole cause of the dynamic illusion effect in this case, it would seem strange that the effects of other illusions also decrease over time (Glover \& Dixon, 2001a, 2001b, 2001c, 2001d).

\section{Extensions of the Planning/Control Model}

In the present study, we have explained the effects of the Ebbinghausillusion on action in terms of a dichotomy between the planning and the on-line control of action. This was also the case for the results of our studies using an orientation illusion (Glover \& Dixon, 2001a, 2001b, 2001d) and a size-contrast illusion (Glover \& Dixon, 2001d). Furthermore, a similar pattern of on-line correction was observed for the effects of printed words on grasping (Glover \& Dixon, 2002b). In all four paradigms, a context-induced optical illusion (or word) had a larger effect on action in the earlier portion of the movement than in the latter portion. Thus, it would appear that the visual information used during planning is more heavily influenced by cognitive and perceptual variables than is on-line control. Indeed, inasmuch as effects of the illusions on control are demonstrably different from their effects on planning and perceptions, the present results represent evidence for a dissociation not just between planning and control, but also between perception and control. Next, we will describe how many other apparent dissociations between perception and action may be better explained as dissociations between perception and control.

One class of dissociation between perception and control is related to the observation that the motor system can accommodate changes in the target that occur outside of conscious awareness. For example, a perturbation in a target's position during a saccade often goes unnoticed, a phenomenon related to saccadic suppression. However, pointing movements directed at the target can compensate accurately for the change in position (Bridgeman et al., 1979; Goodale et al., 1986; Prablanc \& Martin, 1992). Although these studies have been held as evidence for a dissociation between perception and action (Bridgeman et al., 1979; Goodale \& Milner, 1992; Milner \& Goodale, 1995), the adjustments to the new location of the target were being made in flight and, thus, presumably reflected the operation of the on-line control system.

Other results also support a distinction between perception and on-line control. For example, Castiello and Jeannerod (1991) reported that when a target's location was moved coincident with the onset of a reach, participants made adjustments in their trajectories well before they were able to verbally respond to the change (on the order of $150 \mathrm{msec}$ for motor adjustments vs. $300 \mathrm{msec}$ for verbal response). In fact, participants reported having the sensation that they had adjusted the trajectories of their reaches before they had become aware of the change in the position of the target. Similarly, a subtle change in the size of an approaching target that goes unnoticed by participants can be quickly and accurately accommodated by the motor system (Savelsbergh, Whiting, \& Bootsma, 1991). Because these motor adjustments occur during the movements themselves, they would seem to imply a specific dissociation between perception and on-line control, rather than a more general dissociation between perception and action. In contrast to this evidence for different visual information's being used for perception and control, support for the notion that the planning of movements is dissociable from perception is much less evident.

However, it is necessary to point out that the planning/ control model may not account as well for eye movements as it does for other types of movements. For example, Wong and Mack (1981) observed that participants were able to accurately perform saccadic eye movements to a target despite illusory motion induced by a shift in a frame surrounding the target (Roelef's effect). Crucially, the saccades made by the participants in this study did not originally go in the direction of the perceived motion, as would be predicted by the planning/control model. Rather, the saccades moved accurately to the target location.

We suggest that this result may occur because eye and limb movements operate under very different constraints. On the one hand, saccades involve the two-dimensional rotation of the eyes in the orbit and have no need to take into account any obstacles or environmental affordances. On the other hand, a body movement (of which reaching and grasping is but one example) generally involves a three-dimensional trajectory through physical space and must account for obstacles and affordances. Thus, the context of the target may be much less important for the planning of saccades than it is for the planning of body movements, and as a consequence, context-inducedillusions may have little effect on saccadic planning. Consistent with this distinction between eye and body movements, there is evidence that the neural structures underlying the two types of movements are differently localized (Anderson et al., 1994; Deiber, Ibanez, Sadato, \& Hallett, 1996) and rely on separate efferent pathways (Kolb \& Whishaw, 1995).

\section{Conclusions}

The present study revealed a dynamic effect of the Ebbinghaus illusion on grasping. This effect occurred whether or not vision was available during the reach. These results support the planning/control model of action; in this model, planning uses a context-dependent visual representation, whereas control uses a context-independent representation. As such, context-inducedoptical illusions should affect the planning of an action, but not its subsequent control. In contrast, the results of the present study do not support a perception/action model, in which actions are both planned and controlled using a visual representation that is context independent.

The planning/control model provides a compelling explanation of the effects of context-inducedillusions on actions. It can also accommodate many of the findings that have typically been interpreted as dissociations between perception and action. In sum, we believe that the plan- 
ning/control model provides a valuable framework for interpreting a range of phenomena related to the organization and function of the motor system.

\section{REFERENCES}

Abrams, R. A., \& Pratt, J. (1993). Rapid aimed limb movements: Differential effects of practice on component submovements. Journal of Motor Behavior, 25, 288-298.

Aglioti, S., DeSouza, J. F., \& Goodale, M. A. (1995). Size-contrast illusions deceive the eye but not the hand. Current Biology, 5, 679-685.

Anderson, T. J., Jenkins, I., Brooks, D., Hawken, M., Frackowiak, R, \& KenNARD, C. (1994). Cortical control of saccades and fixation in man: A PET study. Brain, 117, 1073-1084.

Bock, O., \& JUNGLING, S. (1999). Reprogramming of grip aperture in a double-step virtual grasping paradigm. Experimental Brain Research, 125, 61-66.

Brenner, E., \& SMeEts, J. (1996). Size illusion influences how we lift but not how we grasp an object. Experimental Brain Research, 111, 473-476.

BRIDGEMAN, B. (1999). Separate representations of visual space for perception and visually guided behavior. In G. Aschersleben, T. Bachmann, \& J. Muessler (Eds.), Cognitive contributions to the perception of spatial \& temporal events (pp. 4-13). Amsterdam: Elsevier.

Bridgeman, B., Gemmer, A., Forsman, T., \& Huemer, V. (2000). Processing spatial information in the sensorimotor branch of the visual system. Vision Research, 40, 3539-3552.

Bridgeman, B., Lewis, S., Heit, G., \& Nagle, M. (1979). Relation between cognitive and motor-oriented systems of visual position perception. Journal of Experimental Psychology: Human Perception \& Performance, 5, 692-700.

Bridgeman, B., Peery, S., \& Anand, S. (1997). Interaction of cognitive and sensorimotor maps of visual space. Perception \& Psychophysics, 59, 456-469.

Castiello, U., Bennett, K., \& Stelmach, G. (1993). Reach to grasp: The natural response to perturbation of object size. Experimental Brain Research, 94, 163-178.

Castiello, U., \& Jeannerod, M. (1991). Measuring time to awareness. NeuroReport, 2, 797-800.

Connolly, J. D., \& Goodale, M. (1999). The role of visual feedback of hand position in the control of manual prehension. Experimental Brain Research, 125, 281-286.

Coren, S., \& Girgus, J. (1978). Seeing is deceiving: The psychology of visual illusions. Hillsdale, NJ: Erlbaum.

Coren, S., \& Miller, J. (1974). Size-contrast as a function of figural similarity. Perception \& Psychophysics, 16, 355-357.

Daprati, E., \& Gentilucci, M. (1997). Grasping an illusion. Neuropsychologia, 35, 1577-1582.

Deiber, M.-P., Ibanez, V., S ADAto, N., \& Hallett, M. (1996). Cerebral structures participating in motor preparation in humans: A positron emission tomography study. Journal of Neurophysiology, 75, 233-247.

Dixon, P. (1998). Why scientists value $p$ values. Psychonomic Bulletin \& Review, 5, 390-396.

Dixon, P., \& O'Reilly, T. (1999). Scientific versus statistical inference. Canadian Journal of Experimental Psychology, 53, 133-149.

EKLUND, G. (1972). Position sense and state of contraction. Journal of Neurology, Neurosurgery, \& Psychiatry, 35, 606-611.

Elliot, D., Binsted, G., \& HeAth, M. (1999). The control of goaldirected movements: Correcting errors in the trajectory. Human Movement Science, 18, 121-136.

EvarTs, E. V., \& VAUGHN, W. (1978). Intended arm movements in response to externally produced arm displacements in man. In J. E. Desmedt (Ed.), Cerebral motor control in man: Long loop mechanisms (Progress in Clinical Neurophysiology,pp. 178-192). Basel: Karger.

Flash, T., \& Henis, E. (1991). Arm trajectory modifications during reaching towards visual targets. Journal of Cognitive Neuroscience, $\mathbf{3}$, 220-230.

Franz, V. H., Gegenfurtner, K., Bülthoff, H., \& Fahle, M. (2000). Grasping visual illusions: No evidence for a dissociation between perception and action. Psychological Science, 11, 20-25.
Gentilucci, M., Chieffi, S., Daprati, E., Saetti, M., \& Toni, I. (1996). Visual illusion and action. Neuropsychologia, 34, 369-376.

Gentilucci, M., Toni, I., Chieffi, S., \& PaVesi, G. (1994). The role of proprioception in the control of prehension movements: A kinematic study in a peripherally deafferented patient and in normal subjects. Experimental Brain Research, 99, 483-500.

GLOVER, S. (2001). Separate visual representations in the planning and control of action: Studies in healthy and brain-damaged populations. Manuscript submitted for publication.

Glover, S., \& Dixon, P. (2001a). Dynamic illusion effects in a reaching task: Evidence for separate visual representations in the planning and control of reaching. Journal of Experimental Psychology: Human Perception \& Performance, 27, 560-572.

Glover, S., \& Dixon, P. (2001b). Motor adaptation to an optical illusion. Experimental Brain Research, 137, 254-258.

Glover, S., \& Dixon, P. (2001c). The Müller-Lyer illusion and the accuracy of lower limb movements. Manuscript in preparation.

Glover, S., \& Dixon, P. (2001d). The role of vision in the on-line correction of illusion effects on action. Canadian Journal of Experimental Psychology, 55, 96-103.

Glover, S., \& Dixon, P. (2002a). The importance of contour discrimination in the on-line correction of illusion effects on action. Manuscript in preparation.

Glover, S., \& Dixon, P. (2002b). Semantics affect the planning but not on-line control of grasping. Manuscript submitted for publication.

Goodale, M. A., \& Milner, A. (1992). Separate visual pathways for perception and action. Trends in Neurosciences, 15, 20-25.

Goodale, M. A., Pelisson, D., \& Prablanc, C. (1986). Large adjustments in visually guided reaching do not depend on vision of the hand or perception of target displacement. Nature, 320, 748-750.

Goodman, S. N., \& Royall, R. (1988). Evidence and scientific research. American Journal of Public Health, 78, 1568-1574.

Haffenden, A. M., \& Goodale, M. (1998). The effect of pictorial illusion on prehension and perception. Journal of Cognitive Neuroscience, 10, 122-136.

HagGard, P., \& Wing, A. (1990). Assessing and reporting the accuracy of position measurements made with optical tracking systems. Journal of Motor Behavior, 22, 315-321.

Haggard, P., \& WING, A. (1997). On the hand transport component of prehensile movements. Journal of Motor Behavior, 29, 282-287.

JACKson, G. M., JaCkson, S., Husain, M., Harvey, M., Kramer, T., \& Dow, L. (2000). The coordination of bimanual prehension movements in a centrally deafferented patient. Brain, 123, 380-393.

JACKSON, S. R., \& SHAW, A. (2000). The Ponzo illusion affects grip force but not grip aperture scaling during prehension movements. Journal of Experimental Psychology: Human Perception \& Performance, 26, 418-423.

JAKOBSON, L. S., \& GoOdALE, M. (1991). Factors affecting higher-order movement planning: A kinematic analysis of human prehension. $E x$ perimental Brain Research, 86, 199-208.

JEANNEROD, M. (1984). The timing of natural prehension movements. Journal of Motor Behavior, 16, 235-254.

JEANNEROD, M. (1988). The neural and behavioural organization of goal-directed movements. Oxford: Oxford University Press.

Jeannerod, M., Michel, F., \& Prablanc, C. (1984). The control of hand movements in a case of hemianaesthesia following a parietal lesion. Brain, 107, 899-920.

Keele, S. W., \& Posner, M. (1968). Processing of visual feedback in rapid movements. Journal of Experimental Psychology, 77, 155158.

Khan, M. A., Franks, I., \& Goodman, D. (1998). The effect of practice on the control of rapid aiming movements: Evidence for an interdependency between programming and feedback processing. Quarterly Journal of Experimental Psychology, 51A, 425-444.

Kolb, B., \& WhISHaW, I. (1995). Fundamentals of human neuropsychology. New York: Freeman.

LeE, R. G., \& TATton, W. (1975). Motor responses to sudden limb displacements in primates with specific CNS lesions and in human patients with motor system disorders. Canadian Journal of Neurological Science, 2, 285-293.

Marotta, J. J., DeSouza, J., Haffenden, A., \& Goodale, M. (1998). 
Does a monocularly-presented size-contrast illusion influence grip aperture? Neuropsychologia, 36, 491-497.

Marteniuk, R. G., MacKenzie, C., Jeannerod, M., Athenes, S., \& Dugas, C. (1987). Constraints on human arm movement trajectories. Canadian Journal of Psychology, 41, 365-378.

McCollough, C. (1965). Color adaptation of edge-detectors in the human visual system. Science, 149, 1115-1116.

Meyer, D. E., Abrams, R, Kornblum, S., Wright, C., \& Smith, K. (1988). Optimality in human motor performance: Ideal control of rapid aimed movements. Psychological Review, 95, 340-370.

Milner, A. D., \& Goodale, M. A. (1995). The visual brain in action. Oxford: Oxford University Press.

Mishkin, M., Ungerleider, L., \& MAcko, K. (1983). Object vision and spatial vision: Two cortical pathways. Trends in Neurosciences, $\mathbf{6}$, 414-417.

Otto-de Haart, E. G., Carey, D. P., \& Milne, A. B. (1999). More thoughts on perceiving and grasping the Müller-Lyer illusion. Neuropsychologia, 37, 1437-1444.

Paillard, J., \& Brouchon, M. (1968). Active and passive movements in the calibration of position sense. In S. J. Freedman (Ed.), The neuropsychology of spatially oriented behavior (pp. 37-55). Homewood, IL: Dorsey.

Paulignan, Y., Jeannerod, M., MacKenzie, C., \& Marteniuk, R. (1991). Selective perturbation of visual input during prehension movements: II. The effects of changing object size. Experimental Brain Research, 87, 407-420.

Paulignan, Y., MacKenzie, C., Marteniuk, R, \& Jeannerod, M. (1991). Selective perturbation of visual input during prehension movements: I. The effects of changing object position. Experimental Brain Research, 83, 502-512.

Pavani, F., Boscagli, I., Benvenuti, F., Rabuffetti, M., \& Farne, A. (1999). Are perception and action affected differently by the Titchener circles illusion? Experimental Brain Research, 127, 95-101.

Prablanc, C., \& Martin, O. (1992). Automatic control during hand reaching at undetected two-dimensional target displacements. Journal of Neurophysiology, 67, 455-469.

Pratt, J., \& Abrams, R. (1996). Practice and component submovements: The roles of programming and feedback in rapid aimed limb movements. Journal of Motor Behavior, 18, 396-426.

REDDing, G. M., \& WALLACE, B. (1994). Effects of movement duration and visual feedback on visual and proprioceptive components of prism adaptation. Journal of Motor Behavior, 26, 257-266.

Redding, G. M., \& Wallace, B. (1997). Prism adaptation during target pointing from visible and nonvisible starting locations. Journal of Motor Behavior, 29, 119-130.

Sainburg, R. L., Poizner, H., \& Ghez, C. (1993). Loss of proprioception produces deficits in interjoint coordination. Journal of Neurophysiology, 70, 2136-2147.

Savelsbergh, G. J., Whiting, H., \& Bootsma, R. (1991). Grasping tau. Journal of Experimental Psychology: Human Perception \& Performance, 17, 315-322.

Smeets, J. B., \& Brenner, E. (1995). Perception and action are based on the same visual information: Distinction between position and velocity. Journal of Experimental Psychology: Human Perception \& Performance, 21, 19-31.

Smeets, J. B., Erkelens, C., \& van der Gon, J. (1990). Adjustments of fast goal-directed movements in response to an unexpected inertial load. Experimental Brain Research, 81, 302-312.

STARK, L. (1968). Neurological control systems: Studies in bioengineering. New York: Plenum.

van Donkelaar, P. (1999). Pointing movements are affected by sizecontrast illusions. Experimental Brain Research, 125, 517-520.

Westwood, D. A., Heath, M., \& Roy, E. (2000). The effect of a pictorial illusion on closed-loop and open-loop prehension. Experimental Brain Research, 130, 545-548.

Wing, A. M., \& Fraser, C. (1983). The contribution of the thumb to reaching movements. Quarterly Journal of Experimental Psychology, 35A, 297-309.

Wing, A. M., Turton, A., \& Fraser, C. (1986). Grasp size and accuracy of approach in reaching. Journal of Motor Behavior, 18, 245-260.

Wong, E., \& MAck, A. (1981). Saccadic programming and perceived location. Acta Psychologica, 48, 123-131.

Woodworth, R. S. (1899). The accuracy of voluntary movement. Psychological Review Monographs, 3 (2), 1-114.

ZelazniK, H. N., Hawkins, B., \& Kisselburgh,L. (1983). Rapid visual feedback processing in single-aiming movements. Journal of Motor Behavior, 15, 217-236.

(Manuscript received October 30, 2000; revision accepted for publication May 1, 2001.) 